\title{
Obesity and Poststroke Fatigue: A 2-Year Longitudinal Study
}

\author{
Mengmeng Gu (D) - Lulu Xiao · Jinjing Wang • Qiankun Cai · \\ Yujing Liu $\cdot$ Pengfei Xu $\cdot$ Yuanlu Liu $\cdot$ Xianjun Huang • \\ Wei $\mathrm{Hu} \cdot$ Wen Sun
}

Received: July 15, 2021 / Accepted: August 17, 2021 / Published online: August 26, 2021

(C) The Author(s) 2021

\section{ABSTRACT}

Introduction: Obesity is associated with fatigue in many diseases, but the correlation between obesity and poststroke fatigue (PSF) is unclear. The study aimed to investigate the relationship between body mass index (BMI) and the occurrence of and recovery from PSF.

Methods: Within 3 days of the onset of ischemic stroke, patients were consecutively recruited. We assessed PSF at admission and at

Mengmeng Gu, Lulu Xiao and Jinling Wang contributed equally to this work.

Supplementary Information The online version contains supplementary material available at https:// doi.org/10.1007/s40120-021-00276-X.

M. Gu

Department of Neurology, Nanjing First Hospital, Nanjing Medical University, Nanjing, China

L. Xiao $\cdot$ J. Wang

Department of Neurology, Affiliated Jinling

Hospital, Medical School of Nanjing University,

Nanjing, China

Q. Cai

Department of Neurology, The Second Affiliated

Hospital of Fujian Medical University, Quanzhou, China

Y. Liu

Department of Neurology, Jinling Hospital, Nanjing

Medical University, Nanjing, China
6, 12, and 24 months with the Fatigue Severity Scale (FSS). Multivariable logistic regression and restricted cubic spline function were used to explore the relationships between baseline BMI and the risk of PSF at different time points. The correlation between baseline BMI and the dynamic changes in the natural logarithm transformation of the FSS (lnFSS) score during the follow-up period was analyzed by the piecewise linear mixed-effects model.

Results: A total of 1026 stroke patients were included. Multivariable analyses indicated that obesity was associated with a decreased risk of early PSF (fatigue diagnosed at baseline) [odds ratio (OR) $0.61,95 \%$ confidence interval (CI) $0.41-0.93$ ] but an increased risk of late PSF (fatigue diagnosed 6 months after the index

\footnotetext{
P. Xu $\cdot$ W. Hu $(\varangle) \cdot$ W. Sun $(\varangle)$

Stroke Center and Department of Neurology, The

First Affiliated Hospital of USTC, Division of Life

Sciences and Medicine, University of Science and

Technology of China, Hefei, Anhui, China

e-mail: andinghu@126.comW. Sun

e-mail: sunwen_medneuro@163.com

Y. Liu

Department of Neurology, Jinling Hospital, The First

School of Clinical Medicine, Southern Medical

University, Nanjing, China

X. Huang

Department of Neurology, Yijishan Hospital,

Wannan Medical College, Wuhu, Anhui Province,

China
} 
stroke) (OR 1.63, 95\% CI 1.06-2.50 for 6 months; OR 1.87, 95\% CI 1.18-2.96 for 12 months; OR 2.11, 95\% CI 1.28-3.49 for 24 months). Longitudinal analyses indicated that in the late stage of fatigue, the higher the BMI category, the slower the rate of decrease was for the FSS score.

Conclusion: Obese patients are less likely to develop fatigue in the acute stage of ischemic stroke. However, they are more prone to late fatigue and exhibit a slower decline in the FSS score in the long term.

Keywords: Body mass index; Obesity; Fatigue Severity Scale; Poststroke fatigue

\section{Key Summary Points}

Poststroke fatigue (PSF) is a common debilitating symptom that commonly occurs after ischemic stroke and adversely affects the prognosis of stroke.

Obesity is associated with fatigue in many different diseases, but the correlation between obesity and PSF has not been well studied.

Obese patients are less likely to develop fatigue in the acute stage of ischemic stroke; however, they are more prone to late fatigue and exhibit a slower decline in the severity of fatigue.

The correlation between body mass index categories and the risk of PSF or the dynamic changes in the Fatigue Severity Scale score were not completely consistent among the different sex subgroups.

\section{INTRODUCTION}

Poststroke fatigue (PSF) is a common debilitating symptom that commonly occurs after ischemic stroke, with a reported prevalence ranging from 23 to $85 \%$ worldwide [1]. PSF may persist for a long time and negatively affect physical activity [2], functional outcomes [3], social activities [4], and quality of life [5] in stroke survivors. Preventing and managing fatigue is critical for stroke survivors, families, and even society. However, there is currently no effective treatment, in part due to our lack of understanding of its mechanisms and associated risk factors. There is increasing evidence of associations between PSF and cognitive impairment, affective disorders, residual disability, and even biological factors [6, 7]. However, most of these associations are controversial $[8,9]$. Thus, well-designed studies with large cohorts need to be conducted to further expand our understanding of PSF.

Obesity has become a global public health concern, and it directly or indirectly accounts for a large portion of health-related costs [10]. Overweight and obesity were not only important risk factors for stroke, but also related to disability and stroke-related quality of life [11]. In several studies, obesity has been associated with motor fatigue [12], cancer-related fatigue [13-15], chronic fatigue syndrome [16], and fatigue in patients with systemic lupus erythematosus (SLE) [17] and inflammatory bowel diseases [18]. Considering that obesity has been associated with fatigue caused by multiple chronic diseases, we hypothesized that obesity may also be associated with stroke-induced fatigue. However, the link between obesity and PSF has not been thoroughly investigated [19]. Exploring the association between obesity and PSF may be helpful to deepen our understanding of the risk factors for PSF and provide preliminary information for alleviating fatigue symptoms through weight control, as weight is a potentially modifiable factor.

This study is based on a prospective cohort. Our aim was to investigate the relationship between body mass index (BMI) and the risk of PSF as well as determine whether the relationship varies by sex $[20,21]$. In addition, the course of PSF and severity of fatigue fluctuate [22], and the effect of BMI on PSF may also be different in different courses. Therefore, the relationship between baseline BMI and the dynamic changes in the severity of PSF during a 24-month follow-up period was also investigated. 


\section{METHODS}

\section{Study Design and Patients}

This study is a longitudinal, observational cohort conducted in a large-scale general hospital in China to explore the potential predictors for PSF. The inclusion criteria have been reported elsewhere [23]. In brief, patients $\geq$ 18 years old who were diagnosed with acute ischemic stroke according to the ICD-11 within 3 days of onset were consecutively recruited from October 2015 to May 2017. The exclusion criteria were as follows: (1) consciousness disorders, severe aphasia, and hearing or visual loss; (2) cognitive impairment defined as a Mini-Mental State Examination (MMSE) score $\leq 10$, diagnosed by an experienced physician or nurse, or assessed as having inadequate understanding of all the scales used in the study; (3) a history of neurological or psychiatric diseases that cause fatigue (e.g., malignancies, SLE, Parkinson's disease, or multiple sclerosis); (4) consumption of any medication that may cause psychological and emotional changes (including antiepileptic drugs, glucocorticoids, nonsteroidal anti-inflammatory drugs, and antidepressant drugs); (5) the presence of depression or fatigue before the index stroke. Pre-stroke depression was defined as a history of diagnosed depression, use of antidepressants before the index stroke, or a medical record of depression [24, 25]. Patients who reported fatigue lasting $\geq 3$ months before the index stroke were classified as having prestroke fatigue [26].

\section{Standard Protocol Approvals, Registrations, and Patient Consent}

Ethics approval was obtained from the Ethics Committee on Human Experimentation of Jinling Hospital (Nanjing, China) (approval number: 2010NLY-018). Informed consent was obtained from all participants. This study was performed in accordance with the Helsinki Declaration of 1964 and its later amendments.

\section{Baseline Assessments}

For all the patients enrolled, detailed baseline information was collected from the initial interview. All clinical data, related medical histories, neurological functional deficit scale [National Institutes of Health Stroke Scale (NIHSS) score], and the etiology of ischemic stroke [Trial of ORG 10172 in Acute Stroke Treatment (TOAST) criteria] were collected within $24 \mathrm{~h}$ of admission by experienced neurologists (J.W. and Y.L.). The NIHSS score is a widely used neurological deficit grading examination, including the level of consciousness, eye movements, visual fields, facial palsy, motor and sensory impairments, ataxia, language, dysarthria, and neglect [27]. The TOAST criteria include five categories: large artery atherosclerosis, small artery occlusion, cardioembolism, stroke of other determined etiology, and stroke of undetermined etiology [28]. Since BMI is the most pragmatic measure of obesity, which is not only easy to measure in clinical practice, but also conducive to patients' self-monitoring, we used BMI as the classification standard of overweight and obesity. BMI was determined using standardized height and weight measurements (equipment manufacturer: Wuxi Weigher Factory Co., Ltd., model: RGZ-120-RT) and calculated as [weight in $\mathrm{kg}] /[\text { height in } \mathrm{m}]^{2}$. BMI was modeled alternatively as a continuous or categorical variable $[<24=$ underweight (UW) and normal weight (NW); 24-27.9 = overweight; $\geq 28$ = obesity], as suggested for Chinese adults by the Cooperative Meta-analysis Group of China Obesity Task Force in 2002 [29].

The methods used to record neuropsychological data are published elsewhere [23]. In brief, the Chinese version of the 24-item Hamilton Depression Scale (HAMD-24), 14-item Hamilton Anxiety Scale (HAMA-14), and the Lubben Social Network Scale (LSNS) were administered by researchers who underwent the same training to evaluate the symptoms of depression, anxiety, and interpersonal communication, respectively [30-32]. 


\section{Outcomes}

The score of the 9-item Fatigue Severity Scale (FSS), which is the most commonly used fatigue scale, was used as the outcome measure for all patients enrolled [33]. The FSS is a valid and reliable 7-point Likert scale used for assessing and quantifying fatigue in obese patients, with scores ranging from 1 (strongly disagree) to 7 (strongly agree) [34]. A higher score indicates more severe fatigue. The Chinese version of the FSS has been used and validated in the Asian population [35]. In accordance with previous studies, we defined fatigue as an average FSS score $\geq 4$ [36]. Fatigue diagnosed within 6 months of the index stroke was defined as early fatigue, and fatigue diagnosed 6 months and later was defined as late fatigue [23].

The baseline FSS score was recorded within $24 \mathrm{~h}$ of admission. The patients were re-evaluated for fatigue by telephone or face-to-face questionnaires at 6,12 , and 24 months after the index stroke. If a patient had another stroke during the follow-up period, he or she was no longer followed up.

\section{Statistical Analysis}

All analyses were conducted by using Stata 14.1 and SAS 9.4.

Descriptive analyses were performed to examine the characteristics of patients at baseline. Continuous variables are presented as the means [standard deviations (SDs)] or medians (interquartile ranges), and categorical variables are presented as numbers (proportions). The continuous variables and categorical variables were compared by one-way analysis of variance (ANOVA), Wilcoxon rank sum test, and Pearson's chi-square test, as appropriate.

For the cross-sectional analyses of associations between BMI and PSF, multivariable logistic regression models for all patients and patients in different sex subgroups were conducted. In addition to the quantitative relationships between BMI and PSF, we also used restricted cubic splines with 4 knots (at the 5th, 35 th, 65th, and 95th percentiles) to explore the nonlinear relationships between BMI and the risk of PSF. A BMI of $22.5 \mathrm{~kg} / \mathrm{m}^{2}$ has been widely used as the midpoint of the range of normal body weight and was used as the reference point for all subjects (all patients or the patients in different sex subgroups) [37].

For the longitudinal analysis, we used piecewise linear mixed-effects models with random intercepts and slopes to model the relationship between baseline BMI and the FSS score during the 24 months of follow-up. Grand-mean centering was conducted for continuous covariates without meaningful 0 values, which may provide advantages for interpreting the results. Since the FSS score is highly skewed, the natural logarithm transformation is applied (lnFSS). According to previous studies, the course of PSF can be divided into early fatigue and late fatigue periods, with 6 months being used as the cutoff point [23]. Thus, in the piecewise regression model, we set the breakpoint to be 6 months. For all models, we identified the unstructured covariance model to be the best-fit model on the basis of the Akaike information criteria (AIC) [38].

All statistical analyses were conducted with adjustments for the baseline variables: age, sex, hypertension, diabetes mellitus, coronary heart disease, smoking, history of stroke or transient ischemic attack (TIA), baseline NIHSS score, the TOAST criteria, and HAMA, HAMD, and LSNS scores.

\section{Availability of Data and Material}

The datasets used and/or analyzed during the current study are available from the corresponding author on reasonable request.

\section{RESULTS}

Of the 1280 subjects who were screened, 1144 met the aforementioned eligibility criteria and 1026 were eventually prospectively included in the study. Among these patients, 935, 896, and 874 were followed up for 6,12 , and 24 months, respectively. Details on the missing FSS data during the follow-up period can be found in the Appendix (Supplementary Figs. S1 and S2). During the 2-year follow-up period, 77 patients 
failed to complete at least one follow-up. The NIHSS score of these patients was slightly higher than for those with follow-up (Supplementary Table S1).

\section{Cross-Sectional Analyses}

According to BMI, patients were stratified into three groups: UW \& NW, overweight, and obesity. Univariate analysis indicated that there were significant differences in terms of sex $(p=0.038), \quad$ presence of hypertension $(p<0.001)$, presence of diabetes mellitus $(p=0.001)$, baseline NIHSS score $(p=0.028)$, HAMA score $(p=0.006)$, and HAMD score ( $p=0.013$ ) among the three BMI categories. At baseline, the prevalence of PSF was $41.8 \%$. With the extension of follow-up time, the prevalence of PSF gradually decreased (36.0\% for 6 months, $29.8 \%$ for 12 months, and $23.8 \%$ for 24 months). Univariate analysis showed that the prevalence of PSF decreased with the increase of BMI at baseline, but increased with the increase of BMI during follow-up (Table 1).

Multivariable logistic regression indicated that, at baseline, after the model was adjusted for potential confounders, the proportion of obese patients with PSF was significantly lower than that of UW \& NW patients with PSF [odds ratio (OR) $0.61,95 \%$ confidence interval (CI) 0.41-0.93, Table 2 and Fig. 1]. However, during the follow-up period, the proportion of obese patients with PSF was significantly higher than that of UW \& NW patients with PSF at 6, 12, and 24 months (OR 1.63, 95\% CI 1.06-2.50 for 6 months; OR 1.87, 95\% CI 1.18-2.96 for 12 months; OR 2.11, 95\% CI 1.28-3.49 for 24 months). Furthermore, at 12 and 24 months, patients in the overweight category were also significantly more prone to developing PSF than were those in the UW \& NW category (OR 1.50, 95\% CI 1.07-2.11 for 12 months and OR 1.63, 95\% CI 1.11-2.40 for 24 months) (Fig. 1 and Supplementary Table S2).

In the subgroup analysis, the proportion of obese female patients with PSF was found to be significantly lower than that of UW \& NW female patients with PSF at baseline, but this relationship was not observed in males (Table 2 and Supplementary Figure S3). Additional multivariable-adjusted restricted cubic spline regression analyses showed an inverted U-shaped association between BMI and the odds of developing PSF in female patients ( $p$ for nonlinearity $=0.001$ ) but a nearly negative linear relationship ( $p$ for nonlinearity $=0.744$ ) in males at baseline (Fig. 2).

During the follow-up period, the proportion of obese male patients with PSF was significantly higher than that of the UW \& NW male patients with PSF; however, the correlation was not significant in females, regardless of the time point (Supplementary Table S2 and Fig. S3). There were no significant nonlinear relationships between baseline BMI and the proportion of patients with PSF at 6,12 , or 24 months among all patients or the patients in different sex subgroups (Supplementary Fig. S4).

\section{Longitudinal Analyses}

The overall average initial $\operatorname{lnFSS}$ score estimated by the model was 1.054 points (intercept $=1.054, p<0.001)$. The average monthly rate of change in the lnFSS score within the first 6 months was -0.005 (slope $1=-0.005$, $p=0.001)$ and that within the next 18 months was -0.019 (slope $2=-0.019, p<0.001$ ). The variance estimates of the random intercept (intercept $=0.278, p<0.001)$ and random slope of the time variables (slope $1=0.007, p<0.001$; slope $2<0.001, \quad p<0.001)$ were statistically significant, indicating that the initial score and the rate of change in the lnFSS score over time differed significantly among the subjects (Supplementary Table S3).

The initial lnFSS score in the obese group was lower than that in the UW \& NW category (estimate difference $=-0.114, p=0.026$, Table 3 and Fig. 3), but there was no significant difference between the overweight and UW \& NW categories ( $p=0.109$, Table 3 and Fig. 3). After background variables were controlled for, the results of the fixed-effects analysis showed that within the first 6 months, the lnFSS score decreased by 0.018 points per month (estimate $=-0.018, p<0.001$, Table 3 ) in the UW $\&$ NW category (reference), decreased by 0.003 
Table 1 Baseline characteristics of study participants $(N=1026)$

\begin{tabular}{|c|c|c|c|c|c|}
\hline & \multirow[t]{2}{*}{ Total } & \multicolumn{3}{|l|}{ BMI categories } & \multirow[t]{2}{*}{$p$ value } \\
\hline & & $\begin{array}{l}\text { UW \& NW } \\
(N=416)\end{array}$ & $\begin{array}{l}\text { Overweight } \\
(N=463)\end{array}$ & $\begin{array}{l}\text { Obesity } \\
(N=147)\end{array}$ & \\
\hline Age, mean $\pm S D$, years & $61.0 \pm 12.8$ & $61.3 \pm 13.6$ & $61.2 \pm 12.2$ & $59.3 \pm 12.7$ & 0.224 \\
\hline Female, $n(\%)$ & $328(32.0)$ & $140(33.7)$ & $131(28.3)$ & $57(38.8)$ & 0.038 \\
\hline Hypertension, $n$ (\%) & $760(74.1)$ & $275(66.1)$ & $357(77.1)$ & $128(87.1)$ & $<0.001$ \\
\hline Diabetes mellitus, $n$ (\%) & $341(33.2)$ & $113(27.2)$ & $180(38.9)$ & $48(32.7)$ & 0.001 \\
\hline Coronary heart disease, $n(\%)$ & $74(7.2)$ & $33(7.9)$ & $28(6.0)$ & $13(8.8)$ & 0.397 \\
\hline Smoking, $n(\%)$ & $348(33.9)$ & $131(31.5)$ & $170(36.7)$ & $47(32.0)$ & 0.228 \\
\hline Previous stroke or TIA, $n(\%)$ & $279(27.2)$ & $106(25.5)$ & $129(27.9)$ & $44(29.9)$ & 0.528 \\
\hline $\begin{array}{l}\text { Baseline NIHSS score, median } \\
\text { (IQR) }\end{array}$ & $2.0(1.0-6.0)$ & $3.0(1.0-6.0)$ & $2.0(0-6.0)$ & $2.0(1.0-5.0)$ & 0.028 \\
\hline TOAST classification, $n(\%)$ & & & & & 0.145 \\
\hline LAA & 407 (39.7) & $158(38.0)$ & $194(41.9)$ & $55(37.4)$ & \\
\hline SAD & $408(39.8)$ & $168(40.4)$ & $180(38.9)$ & $60(40.8)$ & \\
\hline CE & $112(10.9)$ & $48(11.5)$ & $41(8.9)$ & $23(15.6)$ & \\
\hline Others & $40(3.9)$ & $13(3.1)$ & $24(5.2)$ & $3(2.0)$ & \\
\hline Undetermined & $59(5.8)$ & $29(7.0)$ & $24(5.2)$ & $6(4.1)$ & \\
\hline HAMA score, median (IQR) & $3.0(0-6.0)$ & $2.0(1.0-5.0)$ & $3.0(0-8.0)$ & $4.0(1.0-10.0)$ & 0.006 \\
\hline HAMD score, median (IQR) & $4.0(1.0-9.0)$ & $4.0(1.0-9.0)$ & $4.0(1.0-8.0)$ & $5.0(2.0-12.0)$ & 0.013 \\
\hline LSNS, median (IQR) & $\begin{array}{l}32.0 \\
\quad(22.0-39.0)\end{array}$ & $32.0(19.0-39.0)$ & $31.0(21.0-38.0)$ & $\begin{array}{l}33.0 \\
\quad(26.0-39.0)\end{array}$ & 0.352 \\
\hline $\begin{array}{l}\text { PSF at baseline, } n(\%), \\
\qquad(N=1026)\end{array}$ & $429(41.8)$ & $187(45.0)$ & $188(40.6)$ & $54(36.7)$ & 0.172 \\
\hline $\begin{array}{l}\text { PSF at } 6 \text { months, } n(\%), \\
\qquad(N=935)\end{array}$ & $337(36.0)$ & $115(31.4)$ & $162(37.6)$ & $60(43.5)$ & 0.028 \\
\hline $\begin{array}{l}\text { PSF at } 12 \text { months, } n(\%), \\
\qquad(N=896)\end{array}$ & $267(29.8)$ & $85(23.9)$ & $133(32.4)$ & $49(37.4)$ & 0.005 \\
\hline $\begin{array}{l}\text { PSF at } 24 \text { months, } n(\%), \\
\qquad(N=874)\end{array}$ & $208(23.8)$ & $62(17.7)$ & $105(26.5)$ & $41(32.3)$ & 0.001 \\
\hline
\end{tabular}

$B M I$ body mass index; $U W$ underweight; $N W$ normal weight; $S D$ standard deviation; TIA transient ischemic attack; NIHSS National Institutes of Health Stroke Scale; IQR interquartile range; TOAST Trial of ORG 10172 in Acute Stroke Treatment; $L A A$ large artery atherosclerosis; $S A D$ small artery occlusion; $C E$ cardioembolism; HAMA Hamilton Anxiety Scale; HAMD Hamilton Depression Scale; LSNS Lubben Social Network Scale; PSF poststroke fatigue 
Table 2 Cross-sectional associations between BMI and PSF at baseline

\begin{tabular}{|c|c|c|c|c|c|c|}
\hline & \multicolumn{3}{|c|}{ Univariate model } & \multicolumn{3}{|c|}{ 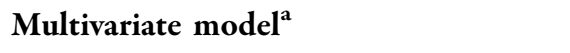 } \\
\hline & OR & $95 \% \mathrm{CI}$ & $p$ value & OR & $95 \% \mathrm{CI}$ & $p$ value \\
\hline \multicolumn{7}{|l|}{ In all patients enrolled } \\
\hline BMI & 0.95 & $0.91-0.99$ & 0.012 & 0.94 & $0.91-0.98$ & 0.007 \\
\hline \multicolumn{7}{|l|}{ BMI categorized as } \\
\hline UW \& NW $(<24)$ & Reference & Reference & Reference & Reference & Reference & Reference \\
\hline Overweight (24-27.9) & 0.84 & $0.64-1.10$ & 0.193 & 0.81 & $0.61-1.07$ & 0.138 \\
\hline Obesity $(\geq 28)$ & 0.71 & $0.48-1.05$ & 0.084 & 0.61 & $0.41-0.93$ & 0.020 \\
\hline \multicolumn{7}{|l|}{ In females $(N=328)$} \\
\hline BMI & 0.93 & $0.87-0.99$ & 0.027 & 0.93 & $0.87-1.01$ & 0.080 \\
\hline \multicolumn{7}{|l|}{ BMI categorized as } \\
\hline UW \& NW $(<24)$ & Reference & Reference & Reference & Reference & Reference & Reference \\
\hline Overweight (24-27.9) & 0.82 & $0.51-1.32$ & 0.404 & 1.02 & $0.57-1.79$ & 0.959 \\
\hline Obesity $(\geq 28)$ & 0.45 & $0.23-0.87$ & 0.017 & 0.40 & $0.18-0.84$ & 0.022 \\
\hline \multicolumn{7}{|l|}{ In males $(N=698)$} \\
\hline BMI & 0.96 & $0.92-1.01$ & 0.150 & 0.95 & $0.90-1.00$ & 0.041 \\
\hline \multicolumn{7}{|l|}{ BMI categorized as } \\
\hline UW \& NW $(<24)$ & Reference & Reference & Reference & Reference & Reference & Reference \\
\hline Overweight (24-27.9) & 0.86 & $0.62-1.19$ & 0.362 & 0.77 & $0.54-1.08$ & 0.128 \\
\hline Obesity $(\geq 28)$ & 0.92 & $0.57-1.49$ & 0.738 & 0.75 & $0.45-1.26$ & 0.283 \\
\hline
\end{tabular}

$B M I$ body mass index; PSF poststroke fatigue; $U W$ underweight; $N W$ normal weight; $O R$ odds ratio; $C I$ confidence interval a All models adjusted for age, sex, hypertension, diabetes mellitus, coronary heart disease, smoking, previous stroke or transient ischemic attack (TIA), baseline National Institutes of Health Stroke Scale (NIHSS) score, Trial of ORG 10172 in Acute Stroke Treatment (TOAST) classification, Hamilton Anxiety Scale (HAMA) score, Hamilton Depression Scale (HAMD) score, and Lubben Social Network Scale (LSNS) score

points per month in the overweight category (estimate $=-0.018+0.015$ ), and increased by 0.027 points per month in the obese category (estimate $=-0.018+0.045)$. The first slopes of the overweight and obese categories were significantly higher than that of the UW \& NW category $(p=0.012$ for overweight; $p<0.001$ for obesity, Table 3). Similar results were observed with the unadjusted model (Supplementary Table S4A). There was also a significant difference in slope 2 among the BMI categories: the higher the BMI category, the flatter slope2 was (Table 3 and Fig. 3).

In the female subgroup, there was no significant difference in the slope between the overweight and UW \& NW categories at all times $(p=0.145$ for slope1; $p=0.402$ for slope2, Supplementary Table S5A and Fig. S5A). However, in the male subgroup, the BMI category affected the overall slope. Specifically, compared with the UW \& NW category, the overweight and obese categories had significantly 


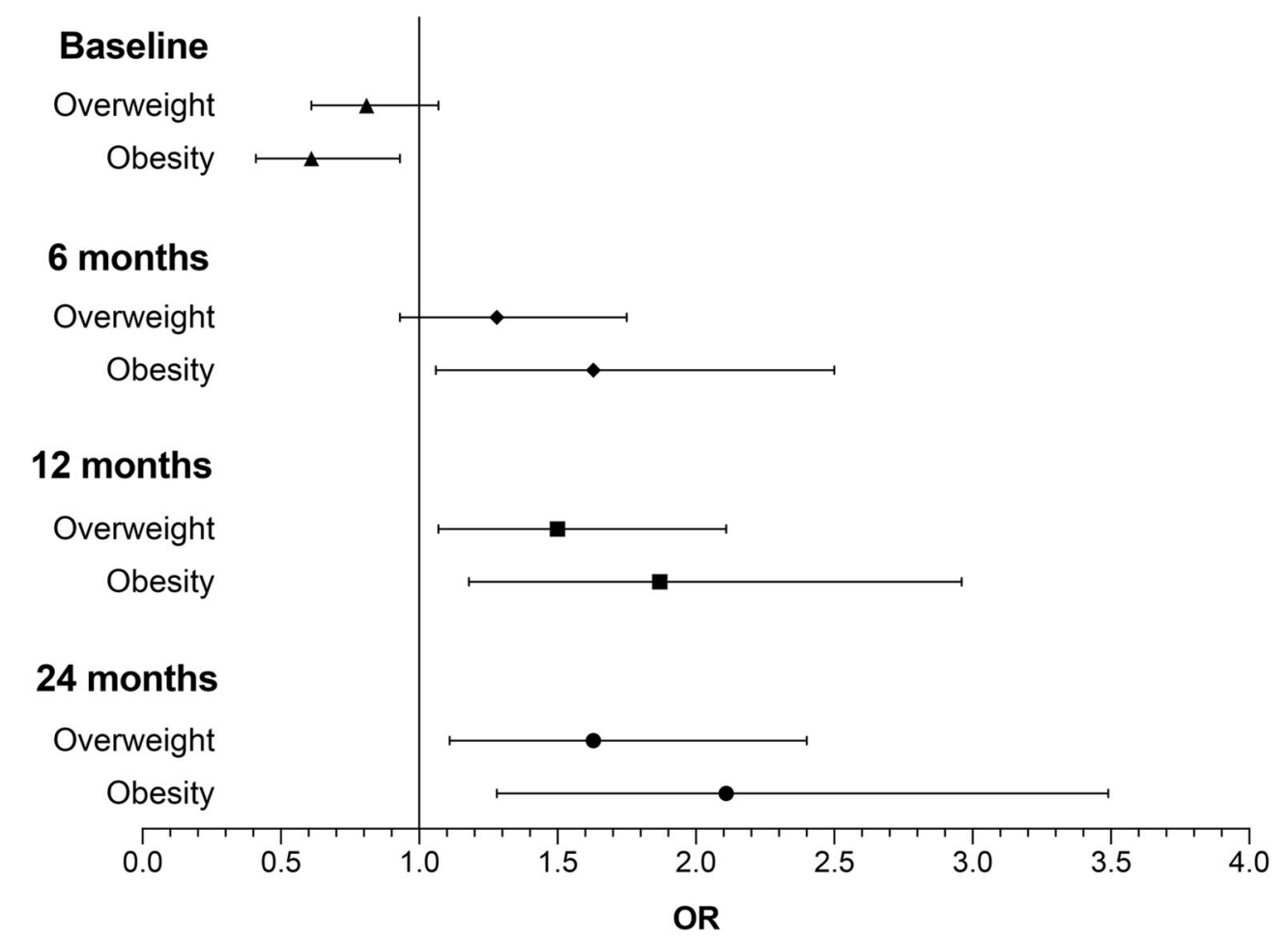

Fig. 1 Cross-sectional associations between BMI and PSF at four time points. BMI body mass index; PSF poststroke fatigue; $O R$ odds ratio

numerically larger slopes (Supplementary Table S5B and Fig. S5B).

\section{DISCUSSION}

This prospective cohort of 1026 stroke patients demonstrated that (1) compared with UW \& NW patients, obese patients were less likely to have early PSF but were more prone to developing late PSF; (2) the FSS score declined at a slower speed in overweight and obese patients than in UW \& NW patients 6 months and later after ischemic stroke; and (3) the correlation between BMI categories and the risk of PSF or the dynamic changes in the FSS score were not completely consistent among the different sex subgroups.

In this study, $41.8 \%(429 / 1026)$ of the patients developed fatigue in the acute phase of ischemic stroke, which is within the reported prevalence of PSF [1]. The prevalence of PSF varies widely depending on the individuals recruited, the time at which fatigue is assessed, and the diagnostic methods used [39]. At the 6-month follow-up, the incidence of PSF decreased to $36.0 \%(337 / 935)$ and continued to decline over the next 18 months $(29.8 \%$ for 12 months and $23.8 \%$ for 24 months), which is consistent with the findings in previous studies that reported a decreasing frequency of PSF over time $[39,40]$. In contrast, in some studies, fatigue remained stable or elevated [41, 42], possibly because of differences in the study population and time points at which fatigue was measured.

Fig. 2 Nonlinear relationship between BMI and PSF at baseline. The nonlinear relationships between BMI and PSF in (A) all patients enrolled, (B) the female subgroup, and $(\mathbf{C})$ male subgroup were modeled by restricted cubic splines. The dotted lines indicate the $95 \%$ confidence intervals of the nonlinear solid line. $x$ axis: continuous BMI in $\mathrm{kg} / \mathrm{m}^{2} \cdot y$ axis: odds ratio (OR) for the estimated PSF. Reference point: $22.5 \mathrm{~kg} / \mathrm{m}^{2}$. BMI body mass index; $P S F$ poststroke fatigue; $O R$ odds ratio 

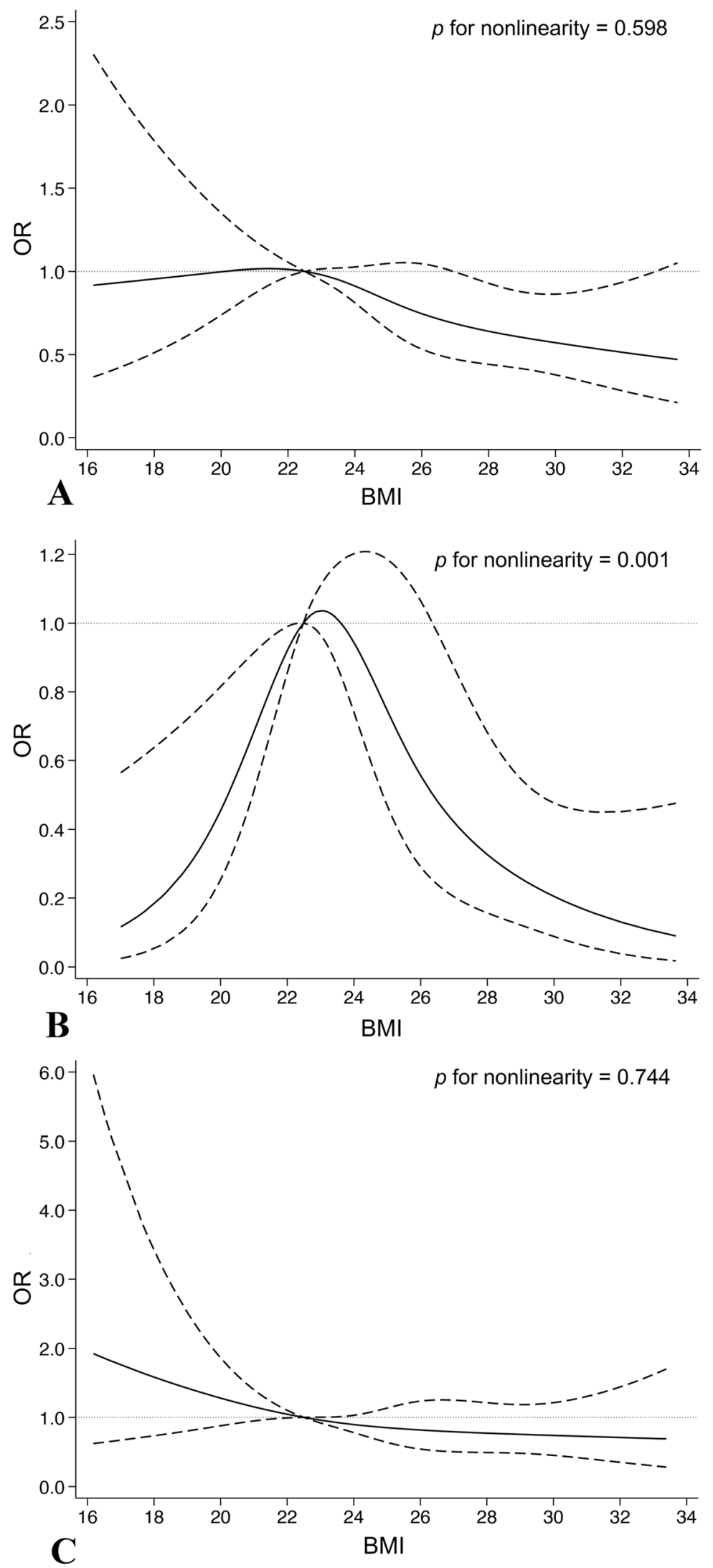
Table 3 Piecewise linear mixed-effects model of the relationship between baseline BMI and lnFSS score during the 24 months of follow-up in all patients enrolled (adjusted model)

\begin{tabular}{|c|c|c|c|}
\hline & Estimate & SE & $p$ value \\
\hline \multicolumn{4}{|l|}{ Fixed effect } \\
\hline Intercept & 0.984 & 0.067 & $<0.001$ \\
\hline \multicolumn{4}{|l|}{ BMI } \\
\hline UW \& NW & Reference & Reference & Reference \\
\hline Overweight & -0.058 & 0.036 & 0.109 \\
\hline Obesity & -0.114 & 0.051 & 0.026 \\
\hline Slope1 (time1) & -0.018 & 0.004 & $<0.001$ \\
\hline \multicolumn{4}{|l|}{ Slope1 * BMI } \\
\hline Slope1 * UW \& NW & Reference & Reference & Reference \\
\hline Slope ${ }^{*}$ overweight & 0.015 & 0.006 & 0.012 \\
\hline Slope1 ${ }^{*}$ obesity & 0.045 & 0.008 & $<0.001$ \\
\hline Slope2 (time2) & -0.022 & 0.001 & $<0.001$ \\
\hline \multicolumn{4}{|l|}{ Slope2 * BMI } \\
\hline Slope2 * UW \& NW & Reference & Reference & Reference \\
\hline Slope ${ }^{*}$ overweight & 0.004 & 0.001 & 0.002 \\
\hline Slope $2 *$ obesity & 0.012 & 0.002 & $<0.001$ \\
\hline \multicolumn{4}{|l|}{ Variance component ${ }^{\mathrm{a}}$} \\
\hline Intercept & 0.262 & 0.012 & $<0.001$ \\
\hline Slope1 (time1) & 0.007 & $<0.001$ & $<0.001$ \\
\hline Slope2 (time2) & $<0.001$ & $<0.001$ & $<0.001$ \\
\hline
\end{tabular}

$B M I$ body mass index; FSS Fatigue Severity Scale; lnFSS score natural logarithm transformed FSS score; $U W$ underweight; $N W$ normal weight; $S E$ standard error

${ }^{a}$ One-tailed test. Adjusted for age, sex, hypertension, diabetes mellitus, coronary heart disease, smoking, previous stroke or transient ischemic attack (TIA), baseline National Institutes of Health Stroke Scale (NIHSS) score, Trial of ORG 10172 in Acute Stroke Treatment (TOAST) classification, Hamilton Anxiety Scale (HAMA) score, Hamilton Depression Scale (HAMD) score, and Lubben Social Network Scale (LSNS) score

Our study found that obese patients are less likely to suffer from early fatigue after ischemic stroke, which is contrary to the conventional understanding and findings reported in previous fatigue-related studies in patients with chronic diseases or healthy controls [13, 43, 44]. However, consistent with our results, Mutai et al. also reported that in patients with acute stroke, BMI is negatively associated with reduced motivation immediately after acute stroke, which was one of the five dimensions of the multidimensional fatigue inventory (MFI) [19]. A possible explanation is that acute stroke produces nutritional challenge, with increased metabolic catabolism due to physiological stress, and reduced caloric intake associated with dysphagia and disability [11]. Stroke patients with high BMI have greater metabolic 


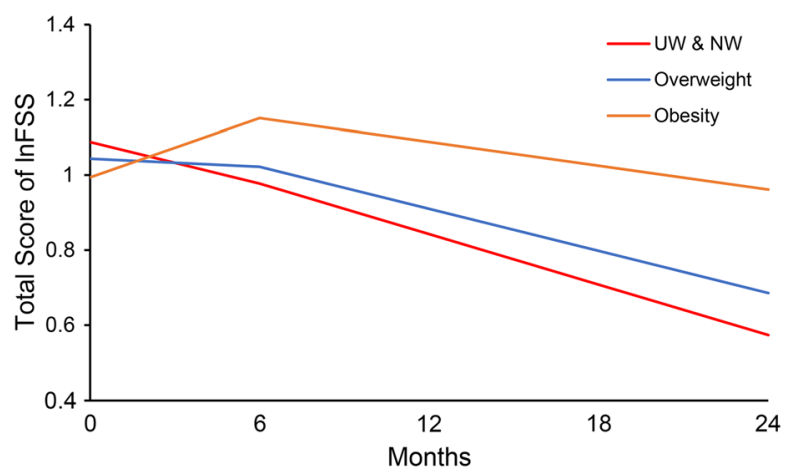

Fig. 3 lnFSS score changes after stroke onset for the three BMI categories during the 24 months of follow-up. The solid line shows the change in the lnFSS score for each BMI category estimated by the piecewise linear mixedeffects model (red for UW \& NW; blue for overweight; orange for obesity). FSS Fatigue Severity Scale; $\ln F S S$ score natural logarithm transformed FSS score; $B M I$ body mass index; $U W$ underweight; $N W$ normal weight

reserve, which may be better able to withstand the nutritional damage $[45,46]$ to maintain the stability of physiological and psychological state. At baseline, UW \& NW patients had higher NIHSS scores, reflecting greater neurological deficits. Previous studies have reported that the nature of stroke itself may be a more important determinant of early fatigue than late fatigue [22]. This might be also one of the reasons why UW \& NW patients are more likely to have early fatigue.

In contrast to the baseline scenario, the FSS score declines more slowly in overweight and obese patients than in UW \& NW patients during the follow-up period. In our study, overweight and obese patients had higher baseline HAMD and HAMA scores. Obese patients are more prone to late fatigue after ischemic stroke, which may reflect the importance of psychological factors in determining late fatigue [22]. This might also be partly explained by the fact that obesity is usually accompanied by more medical comorbidities, exercise disorders, or even cognitive decline, which is likely to lead to the slow recovery of fatigue during a long-term recovery period after ischemic stroke [8, 9, 47, 48]. In addition, overweight and obese patients are more likely to have diabetes mellitus, which increases the risk of late-onset post-stroke depression (PSD) [49]. In view of the high comorbidity of depression and fatigue, late-onset PSD could also affect the recovery of fatigue to a certain extent.

The relationship between BMI and the risk of PSF differed between the sex subgroups. At baseline, an inverted U-shaped curve between BMI and early PSF risk was shown in female patients but not in male patients. During the follow-up period, male but not female obese patients were prone to developing late PSF. The limited literature in this field makes it difficult to postulate the nature of our findings. The possible explanation of gender heterogeneity remains unclear. The effect of discrepancy of hormone secretion on fatigue outcome remains to be systematically investigated.

Although our study is a large sample prospective study with a long follow-up period, it has all the typical limitations inherent in any single-center prospective analysis. First and most importantly, BMI was measured only once at baseline. We did not assess BMI at the followups, mostly because the purpose of this study was to determine whether baseline BMI can be used to predict PSF risk and FSS dynamics; moreover, there is inevitable bias in self-reported weight change determined via telephone interviews. Additionally, according to previous studies, patients with non-severe stroke are less likely to experience changes in weight [50], and the NIHSS scores of the patients included in this study were low. Hence, the correlation between weight changes and the risk of PSF was not considered. Second, BMI is the most pragmatic measure of obesity and is easy to measure in clinical practice, but it cannot distinguish between fat and muscle mass. Therefore, indicators such as the waist circumference, waist-tohip ratio, and body fat mass need to be included in future studies. Third, participants completed face-to-face or telephone questionnaires during three follow-up times. Different data collection methods may affect the results. Fourth, participation in physical activity and rehabilitation may also have an impact on the degree of fatigue of patients, which needs to be evaluated in subsequent studies. Finally, patients with communication difficulties and severe cognitive impairment were excluded because of the need 
to complete multiple neuropsychological scales, and the baseline NIHSS score of patients included in the study was relatively low. Thus, the results of this study cannot be extended to all ischemic stroke populations.

\section{CONCLUSIONS}

Our study suggested that obese patients are less likely to develop fatigue in the acute stage of ischemic stroke. However, they are more prone to developing late fatigue and have a slower decline in the FSS score in the long term.

\section{ACKNOWLEDGEMENTS}

We are deeply grateful to the patients for their long-term participation in this study. We thank Min Li, Dezhi Liu, Yunfei Han, and Yongjun Jiang for their help with data collection.

Funding. Funding for this study, including funding the journal's Rapid Service, was provided in part by Fundamental Research Funds for the Central Universities (grant number WK9110000056) and Natural Science Foundation of Anhui Province (No. 2008085QH368, 2108085MH271).

Authorship. All named authors meet the International Committee of Medical Journal Editors (ICMJE) criteria for authorship for this article, take responsibility for the integrity of the work as a whole, and have given their approval for this version to be published.

Authors' Contributions. MG, LX, and WS contributed to study concept and design. MG and QC contributed to analysis of data. JW, YL, $\mathrm{PX}, \mathrm{YL}$, and $\mathrm{XH}$ contributed to study design and acquisition data. MG, QC, and PX drafted figures of the manuscript. WH and WS drafted and revised the manuscript. All authors revised the manuscript and approved the final version to be published.

Disclosures. Mengmeng Gu, Lulu Xiao, Jinjing Wang, Qiankun Cai, Yujing Liu, Pengfei
$\mathrm{Xu}$, Yuanlu Liu, Xianjun Huang, Wei Hu, and Wen Sun declare that they have nothing to disclose.

Compliance with Ethics Guidelines. This study was performed in accordance with the Helsinki Declaration of 1964 and its later amendments. Ethics approval was obtained from the Ethics Committee on Human Experimentation of Jinling Hospital (Nanjing, China) (approval no. 2010NLY-018). Informed consent was obtained from all participants.

Data Availability. The datasets generated during and/or analyzed during the current study are available from the corresponding author on reasonable request.

Open Access. This article is licensed under a Creative Commons Attribution-NonCommercial 4.0 International License, which permits any non-commercial use, sharing, adaptation, distribution and reproduction in any medium or format, as long as you give appropriate credit to the original author(s) and the source, provide a link to the Creative Commons licence, and indicate if changes were made. The images or other third party material in this article are included in the article's Creative Commons licence, unless indicated otherwise in a credit line to the material. If material is not included in the article's Creative Commons licence and your intended use is not permitted by statutory regulation or exceeds the permitted use, you will need to obtain permission directly from the copyright holder. To view a copy of this licence, visit http://creativecommons.org/licenses/by$\mathrm{nc} / 4.0 /$.

\section{REFERENCES}

1. Cumming TB, Packer M, Kramer SF, English C. The prevalence of fatigue after stroke: a systematic review and meta-analysis. Int J Stroke. 2016;11(9): 968-77.

2. Thilarajah S, Mentiplay BF, Bower KJ, Tan D, Pua YH, Williams $\mathrm{G}$, et al. Factors associated with poststroke physical activity: a systematic review and 
meta-analysis. Arch Phys Med Rehabil. 2018;99(9): 1876-89.

3. Maaijwee NA, Arntz RM, Rutten-Jacobs LC, Schaapsmeerders P, Schoonderwaldt HC, van Dijk EJ, et al. Post-stroke fatigue and its association with poor functional outcome after stroke in young adults. J Neurol Neurosurg Psychiatry. 2015;86(10): 1120-6.

4. Flinn NA, Stube JE. Post-stroke fatigue: qualitative study of three focus groups. Occup Ther Int. 2010;17(2):81-91.

5. Bullier B, Cassoudesalle $\mathrm{H}$, Villain $\mathrm{M}$, Cogne $\mathrm{M}$, Mollo C, De Gabory I, et al. New factors that affect quality of life in patients with aphasia. Ann Phys Rehabil Med. 2020;63(1):33-7.

6. Paciaroni $M$, Acciarresi $M$. Poststroke fatigue. Stroke. 2019;50(7):1927-33.

7. MacIntosh BJ, Edwards JD, Kang M, Cogo-Moreira H, Chen JL, Mochizuki G, et al. Post-stroke fatigue and depressive symptoms are differentially related to mobility and cognitive performance. Front Aging Neurosci. 2017;9:343.

8. Lagogianni C, Thomas S, Lincoln N. Examining the relationship between fatigue and cognition after stroke: a systematic review. Neuropsychol Rehabil. 2018;28(1):57-116.

9. Mead GE, Graham C, Dorman P, Bruins SK, Lewis SC, Dennis MS, et al. Fatigue after stroke: baseline predictors and influence on survival. Analysis of data from UK patients recruited in the International Stroke Trial. PLoS ONE. 2011;6(3): e16988.

10. Collaboration NCDRF. Trends in adult body-mass index in 200 countries from 1975 to 2014: a pooled analysis of 1698 population-based measurement studies with 19.2 million participants. Lancet. 2016;387(10026):1377-96.

11. Liu Z, Sanossian N, Starkman S, Avila-Rinek G, Eckstein M, Sharma LK, et al. Adiposity and outcome after ischemic stroke: obesity paradox for mortality and obesity parabola for favorable functional outcomes. Stroke. 2020. https://doi.org/10. 1161/STROKEAHA.119.027900.

12. Mehta RK. Impacts of obesity and stress on neuromuscular fatigue development and associated heart rate variability. Int $J$ Obes (Lond). 2015;39(2): 208-13.

13. Gerber LH. Cancer-related fatigue: persistent, pervasive, and problematic. Phys Med Rehabil Clin N Am. 2017;28(1):65-88.
14. Inglis JE, Janelsins MC, Culakova E, Mustian KM, Lin PJ, Kleckner IR, et al. Longitudinal assessment of the impact of higher body mass index on cancerrelated fatigue in patients with breast cancer receiving chemotherapy. Support Care Cancer. 2020;28(3):1411-8.

15. Vissers PAJ, Martucci RB, Mols F, Bours MJL, Winkels RM, Kampman E, et al. The impact of body mass index and waist circumference on health-related quality of life among colorectal cancer survivors: results from the PROFILES registry. Nutr Cancer. 2017;69(8):1177-84.

16. Flores S, Brown A, Adeoye S, Jason LA, Evans M. Examining the impact of obesity on individuals with chronic fatigue syndrome. Workplace Health Saf. 2013;61(7):299-307.

17. Patterson SL, Schmajuk G, Jafri K, Yazdany J, Katz P. Obesity is independently associated with worse patient-reported outcomes in women with systemic lupus erythematosus. Arthritis Care Res (Hoboken). 2019;71(1):126-33.

18. Jain A, Nguyen NH, Proudfoot JA, Martin CF, Sandborn WJ, Kappelman MD, et al. Impact of obesity on disease activity and Patient-Reported Outcomes Measurement Information System (PROMIS) in inflammatory bowel diseases. Am J Gastroenterol. 2019;114(4):630-9.

19. Mutai H, Furukawa T, Houri A, Suzuki A, Hanihara T. Factors associated with multidimensional aspect of post-stroke fatigue in acute stroke period. Asian J Psychiatr. 2017;26:1-5.

20. Finucane MM, Stevens GA, Cowan MJ, Danaei G, Lin JK, Paciorek CJ, et al. National, regional, and global trends in body-mass index since 1980: systematic analysis of health examination surveys and epidemiological studies with 960 country-years and 9.1 million participants. Lancet. 2011;377(9765): 557-67.

21. Ng M, Fleming T, Robinson M, Thomson B, Graetz $\mathrm{N}$, Margono C, et al. Global, regional, and national prevalence of overweight and obesity in children and adults during 1980-2013: a systematic analysis for the Global Burden of Disease Study 2013. Lancet. 2014;384(9945):766-81.

22. Wu S, Mead G, Macleod M, Chalder T. Model of understanding fatigue after stroke. Stroke. 2015;46(3):893-8.

23. Wang J, Li F, Xiao L, Peng F, Sun W, Li M, et al. Depressed TSH level as a predictor of poststroke fatigue in patients with acute ischemic stroke. Neurology. 2018;91(21):e1971-8. 
24. Kocer E, Kocer A, Degirmenci Y, Eryilmaz M. Longterm depression is a stroke risk factor. Acta Neuropsychiatr. 2011;23(6):292-6.

25. De Ryck A, Fransen E, Brouns R, Geurden M, Peij D, Marien P, et al. Psychosocial problems associated with depression at 18 months poststroke. Int J Geriatr Psychiatry. 2014;29(2):144-52.

26. Lerdal A, Kottorp A. Psychometric properties of the Fatigue Severity Scale-Rasch analyses of individual responses in a Norwegian stroke cohort. Int J Nurs Stud. 2011;48(10):1258-65.

27. Brott T, Adams HP Jr, Olinger CP, Marler JR, Barsan WG, Biller J, et al. Measurements of acute cerebral infarction: a clinical examination scale. Stroke. 1989;20(7):864-70.

28. Adams HP Jr, Bendixen BH, Kappelle LJ, Biller J, Love BB, Gordon DL, et al. Classification of subtype of acute ischemic stroke. Definitions for use in a multicenter clinical trial. TOAST. Trial of Org 10172 in Acute Stroke Treatment. Stroke. 1993;24(1): 35-41.

29. Zhou B, Coorperative Meta-Analysis Group Of China Obesity Task F. Predictive values of body mass index and waist circumference to risk factors of related diseases in Chinese adult population. Zhonghua Liu Xing Bing Xue Za Zhi. 2002;23(1): 5-10.

30. Berkanovic E, Lubben JE, Kitano HH, Chi I. The physical, mental, and social health status of older Chinese: a cross-national study. J Aging Soc Policy. 1994;6(3):73-87.

31. Chen SD, Sun XY, Niu W, Kong LM, He MJ, Fan $\mathrm{HM}$, et al. Correlation between the level of microRNA expression in peripheral blood mononuclear cells and symptomatology in patients with generalized anxiety disorder. Compr Psychiatry. 2016;69: 216-24.

32. Lau CG, Tang WK, Wong KS, Mok V, Ungvari GS. Predictors of the depressive symptomatology of the family caregivers of Chinese stroke patients in Hong Kong. J Psychiatr Ment Health Nurs. 2012;19(4):285-93.

33. Abdulla FA, Al-Khamis FA, Alsulaiman AA, Alshami AM. Psychometric properties of an Arabic version of the Fatigue Severity Scale in patients with stroke. Top Stroke Rehabil. 2019;26(6):448-55.

34. Impellizzeri FM, Agosti F, De Col A, Sartorio A. Psychometric properties of the Fatigue Severity Scale in obese patients. Health Qual Life Outcomes. 2013;11:32.
35. Wang MY, Liu IC, Chiu CH, Tsai PS. Cultural adaptation and validation of the Chinese version of the Fatigue Severity Scale in patients with major depressive disorder and nondepressive people. Qual Life Res. 2016;25(1):89-99.

36. Rosti-Otajarvi E, Hamalainen P, Wiksten A, Hakkarainen T, Ruutiainen J. Validity and reliability of the Fatigue Severity Scale in Finnish multiple sclerosis patients. Brain Behav. 2017;7(7): e00743.

37. Lennon H, Sperrin M, Badrick E, Renehan AG. The obesity paradox in cancer: a review. Curr Oncol Rep. 2016;18(9):56.

38. Lin PD, Cardenas A, Hauser R, Gold DR, Kleinman $\mathrm{KP}$, Hivert MF, et al. Per- and polyfluoroalkyl substances and blood pressure in pre-diabetic adultscross-sectional and longitudinal analyses of the diabetes prevention program outcomes study. Environ Int. 2020;137:105573.

39. Snaphaan L, van der Werf S, de Leeuw FE. Time course and risk factors of post-stroke fatigue: a prospective cohort study. Eur J Neurol. 2011;18(4): 611-7.

40. Christensen D, Johnsen SP, Watt T, Harder I, Kirkevold M, Andersen G. Dimensions of post-stroke fatigue: a two-year follow-up study. Cerebrovasc Dis. 2008;26(2):134-41.

41. Morsund AH, Ellekjaer H, Gramstad A, Reiestad MT, Midgard R, Sando SB, et al. The development of cognitive and emotional impairment after a minor stroke: a longitudinal study. Acta Neurol Scand. 2019;140(4):281-9.

42. Radman N, Staub F, Aboulafia-Brakha T, Berney A, Bogousslavsky J, Annoni JM. Poststroke fatigue following minor infarcts: a prospective study. Neurology. 2012;79(14):1422-7.

43. Correa-Rodriguez M, Mansouri-Yachou JE, CasasBarragan A, Molina F, Rueda-Medina B, AguilarFerrandiz ME. The Association of body mass index and body composition with pain, disease activity, fatigue, sleep and anxiety in women with fibromyalgia. Nutrients. 2019;11(5):1193.

44. Ercan S, Baskurt F, Baskurt Z, Cetin C. Effect of selfperceived fatigue on balance and functional mobility in middle-aged obese women. Acta Med Okayama. 2019;73(2):95-100.

45. Xue Z, Wang Y, Wang L, Shen L, Zhang A, Pan P, et al. Analysis of influencing factors of poststroke depression: is higher body mass index always a risk factor of poststroke depression? J Nerv Ment Dis. 2019;207(3):203-8. 
46. Doehner W, Clark A, Anker SD. The obesity paradox: weighing the benefit. Eur Heart J. 2010;31(2): 146-8.

47. Vantieghem S, Bautmans I, Guchtenaere A, Tanghe A, Provyn S. Improved cognitive functioning in obese adolescents after a 30-week inpatient weight loss program. Pediatr Res. 2018;84(2):267-71.

48. Rhee J, Mehta RK. Functional connectivity during handgrip motor fatigue in older adults is obesity and sex-specific. Front Hum Neurosci. 2018;12:455.
49. Zhang Y, He JR, Liang HB, Lu WJ, Yang GY, Liu JR, et al. Diabetes mellitus is associated with late-onset post-stroke depression. J Affect Disord. 2017;221: 222-6.

50. Jonsson AC, Lindgren I, Norrving B, Lindgren A. Weight loss after stroke: a population-based study from the Lund Stroke Register. Stroke. 2008;39(3): 918-23. 